\title{
Transverse energy flow and charged particle event shapes with ATLAS
}

\section{Deepak KAR ${ }^{* \dagger}$}

(University of Glasgow ( GB))

E-mail: deepak.karecern.ch

The transverse energy flow and charged particle properties provide insights on the structure of hadronic events, for soft collisions as well as events with a hard scale. The observables studied by the ATLAS Collaboration in $\sqrt{s}=7 \mathrm{TeV}$ proton-proton collisions at the LHC include the transverse thrust, thrust minor and transverse sphericity, each defined using the final state charged particles momenta perpendicular to the beam direction. In addition to the differential distributions, the evolution of each event shape variable is presented as a function of the leading charged particle transverse momentum, charged particle multiplicity and summed transverse momentum. The results also includes the sum of the transverse energy of particles as a function of particle pseudorapidity, using calorimetry information. The distributions are compared to the predictions of various Monte Carlo event generators, which generally tend to underestimate the amount of transverse energy at high pseudorapidity.

XXI International Workshop on Deep-Inelastic Scattering and Related Subjects 22-26 April, 2013

Marseilles, France

* Speaker.

On behalf of the ATLAS Collaboration 


\section{Introduction}

Precise measurements of different soft ${ }^{1} \mathrm{QCD}$ processes are necessary to improve the phenomenological models implemented in various Monte Carlo (MC) generators. ATLAS [1] measured event shape and transverse energy flow observables in $7 \mathrm{TeV}$ center-of-mass energy protonproton collisions at the LHC. Event shape observables are a continuous measure of geometric properties of energy flow, sensitive to the transition between non-perturbative to perturbative regime. Transverse energy flow uses the full acceptance of the calorimeter, and provides a complimentary way of looking at soft particle production from the usual underlying event and minimum bias measurements.

\section{Event shape observables}

ATLAS measured transverse thrust, transverse thrust minor and transverse sphericity requiring at least 6 charged particles with transverse momentum $p_{\mathrm{T}}>0.5 \mathrm{GeV}$ and pseudorapidity $|\eta|<2.5$ in each event [2]. These observables are defined such that high values indicate more spherical events, while the low values represent more dijet-like event topology.

In Fig. 1, the distribution of the three event shape variables are shown in different ranges of the leading track $p_{\mathrm{T}}$. For the inclusive distributions, a prevalence of spherical events is seen, and Pythia 6 [3] tune Z1 [4] describes the data the best. As the leading track $p_{\mathrm{T}}$ is increased, the transition of transverse sphericity to less spherical events can be observed, while at this range transverse thrust and thrust minor are insensitive to that. The tunes optimized to describe the underlying event activity better predict the data for these distributions. In Fig. 2, the mean values of the event shape variables as functions of charged particle multiplicity and $\sum p_{\mathrm{T}}$ are shown. The mean values are seen increase to values consistent with the peak positions of the differential distributions. Generally the MC models describe the data better for the mean values than for differential distributions.

\section{Transverse energy flow}

The measurement of $\sum E_{\mathrm{T}}$, which is defined as the sum of $E_{\mathrm{T}}$ of all stable charged particles with momentum $p>0.5 \mathrm{GeV}$ and all stable neutral particles with $p>0.2 \mathrm{GeV}$, was performed in ATLAS events with minimum bias and dijet topology [5]. The minimum bias analysis contained at least two charged particles with with $p_{\mathrm{T}}>0.5 \mathrm{GeV}$ and $|\eta|<2.5$. The dijet selection required at least two jets with $E_{\mathrm{T}}^{\text {jet }}>20 \mathrm{GeV}$ and $\left|\eta^{\text {jet }}\right|<2.5$, reconstructed with the anti- $k_{t}$ algorithm with radius parameter $R=0.4$. In order to select a well balanced back-to-back dijet system, the jets were required have an azimuthal angular difference of at least 2.5 radians, and the subleading jet had to have at least half of the energy of the leading jet.

In Fig. 3, the inclusive distribution of $\sum E_{\mathrm{T}}$ in these two topologies are shown as a function of $|\eta|$, as well as their ratio. Lower momentum particles in the central region produces a rise, and it can be seen from the ratio that dijet events are more energetic. EPOS [6] and PYTHIA 6 tune AMBT1 [7] describe the data the best, but the agreement gets worse in forward regions. This can

\footnotetext{
${ }^{1}$ By soft we mean low transverse momenta transfer from initial to final state and very few or no particles produced with significant $p_{\mathrm{T}}$.
} 

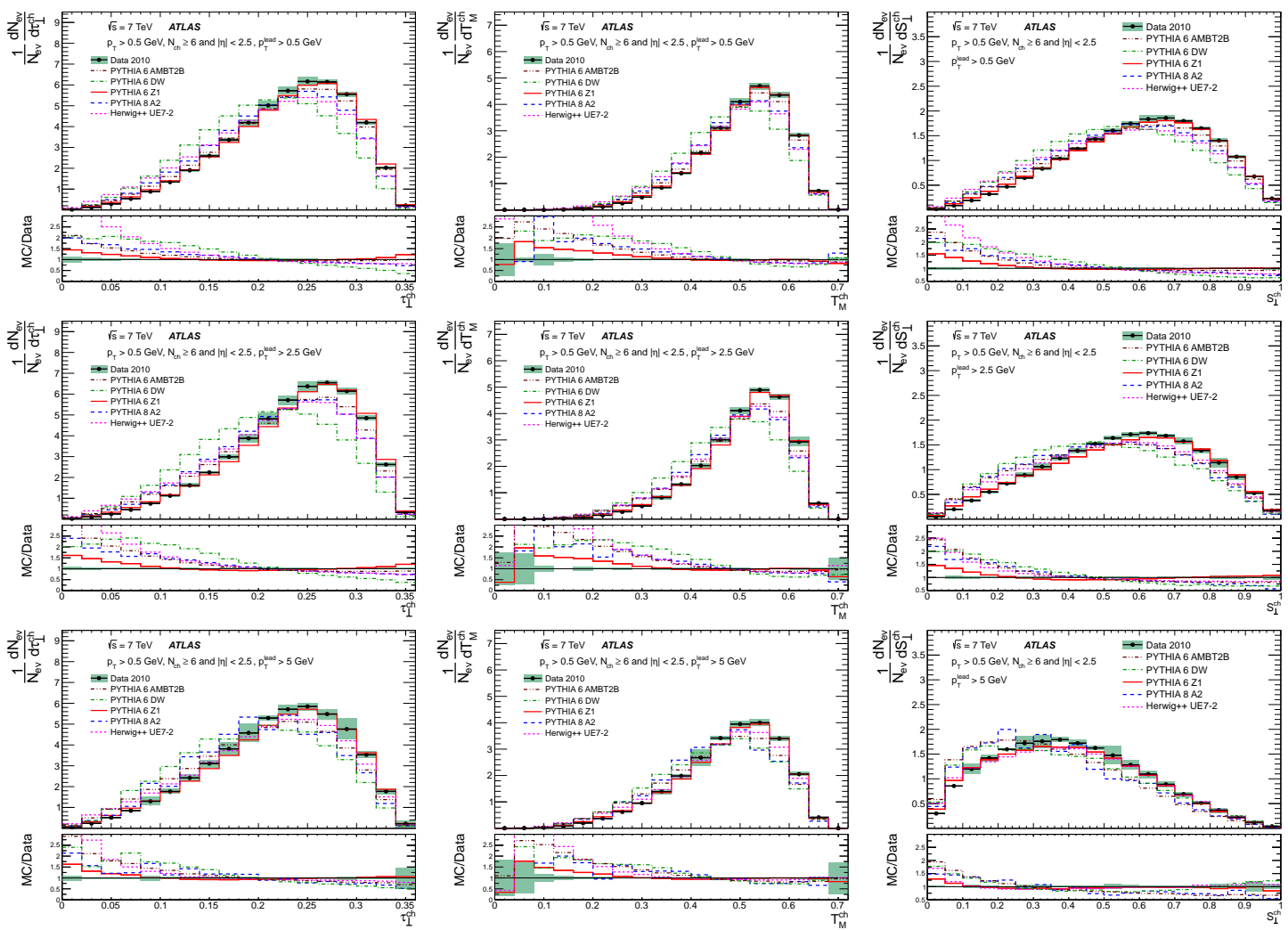

Figure 1: The distributions of transverse thrust (left column), transverse thrust minor (middle column) and transverse sphericity (right column) are shown for different values of minimum $p_{\mathrm{T}}$ of the leading track, with the data compared to different MC predictions [2].

be more clearly seen in Fig. 4, where the $\sum E_{\mathrm{T}}$ distributions in different $|\eta|$ ranges are shown for both toplogies. All MC predictions do better in central region but underestimate the data at high pseudorapidity for the minimum bias topology, while for dijet events, EPOS does not do well in the central region, and dedicated underlying event tunes do better.

ATLAS has previously looked at the effect of parton density function (PDF) changes on tunes [8]. In Fig. 5, the effect of changing the PDF for PythiA 8 [9] tune A2 [8] tune is shown. The A2 tune obtained with CTEQ6L1 [10] PDF performs worse than the same tune obtained using MSTW 2008 LO [11] PDF. If the tune obtained with CTEQ6L1 is used with MSTW 2008 LO PDF, a slight improvement can be observed - which can be attributed to more high and low-x gluons in MSTW 2008 LO PDF.

\section{Summary}

While these measurements show the inadequacy of the MC models at the LHC energy regime, they are crucial input to MC tuning as we move to higher LHC centre-of-mass energies. Modelling the energy flow in forward region is critical for many measurements and searches. 

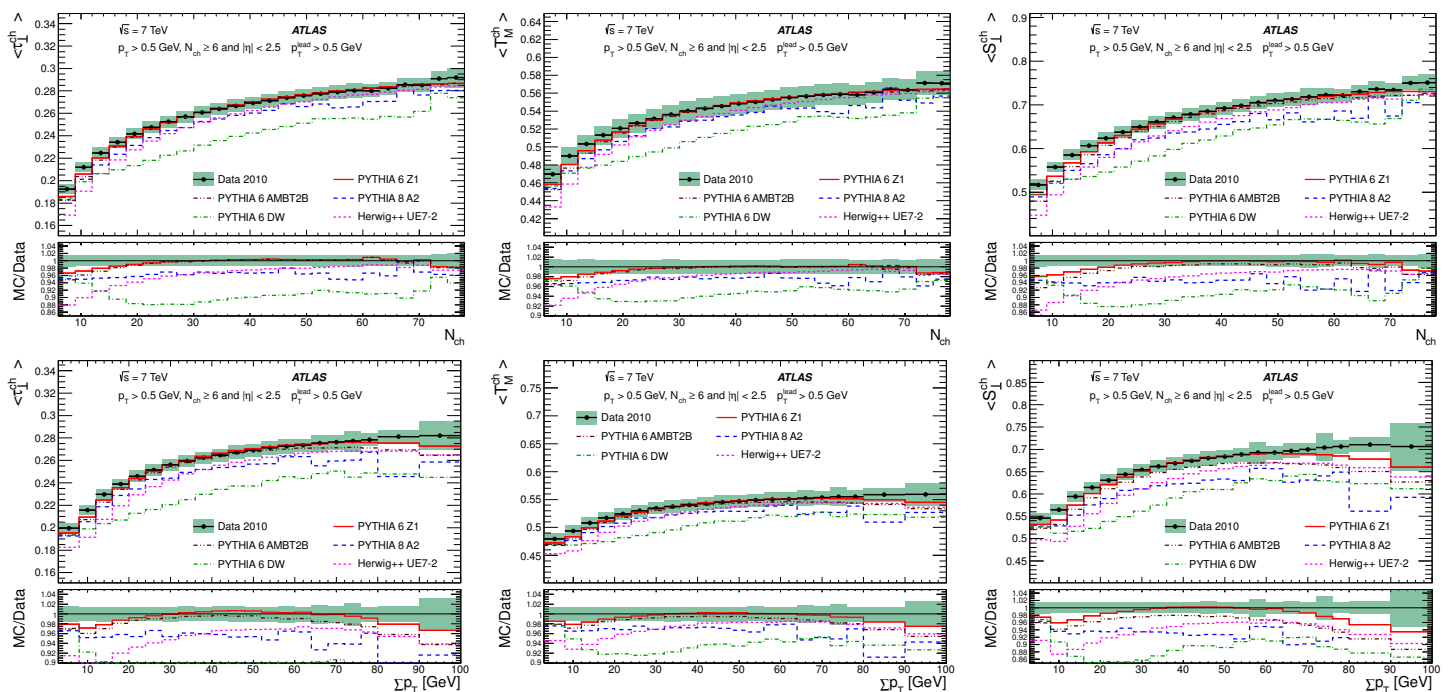

Figure 2: The distributions of transverse thrust (left column), transverse thrust minor (middle column) and transverse sphericity (right column) as function of number of charged particles (top row) and charged particle sum $p_{\mathrm{T}}$ (bottom row), with the data compared to different MC predictions [2].
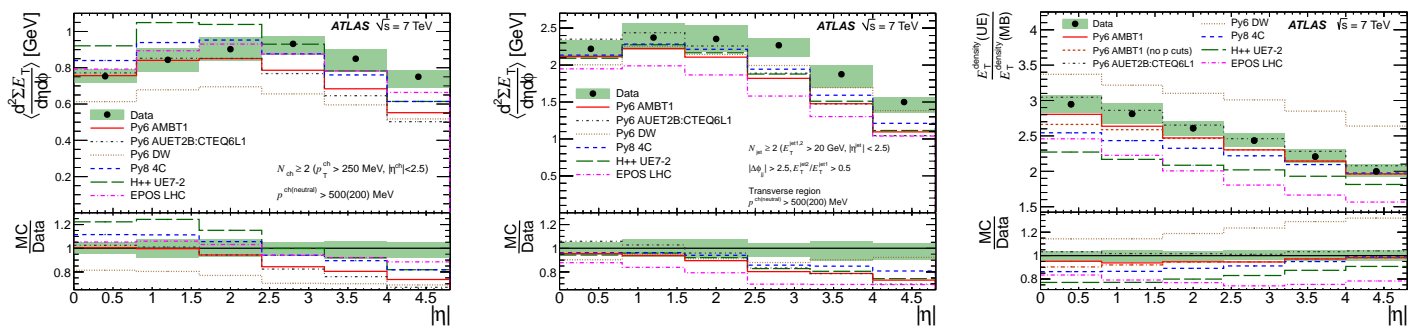

Figure 3: The distribution of transverse energy flow as a function of pseudorapidity are shown in minimum bias (left) and dijet (middle) event selections. The ratio is shown on the right. The data are compared with different MC predictions [5].

\section{References}

[1] ATLAS Collaboration, The ATLAS Experiment at the CERN Large Hadron Collider, JINST 3 (2008) S08003.

[2] The ATLAS Collaboration, arXiv:1207.6915 [hep-ex]. submitted to PRD, 2012.

[3] T. Sjostrand, S. Mrenna, and P. Skands, PYTHIA 6.4 physics and manual, JHEP 05 (2006) 026, hep-ph/0603175.

[4] R. Field, Acta Phys. Polon. B 42, 2631 (2011) [arXiv:1110.5530 [hep-ph]].

[5] The ATLAS Collaboration, JHEP 1211, 033 (2012) [arXiv:1208.6256 [hep-ex]].

[6] S. Porteboeuf, T. Pierog, and K. Werner, Producing Hard Processes Regarding the Complete Event: The EPOS Event Generator, arXiv:1006.2967 [hep-ph].

[7] The ATLAS Collaboration, Charged particle multiplicities in inelastic pp interactions with ATLAS and the ATLAS Minimum Bias Tune 1, ATLAS-CONF-2010-031, 2010. 

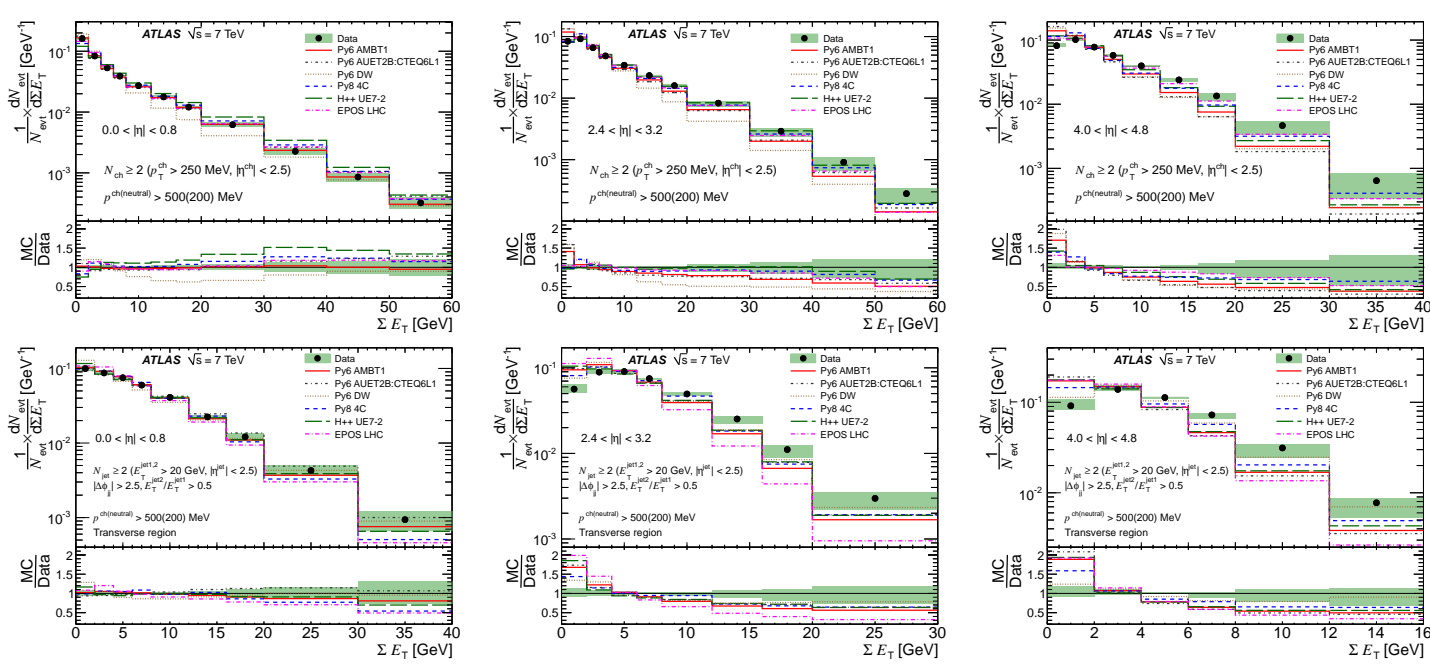

Figure 4: The distribution of transverse energy flow is shown for different pseudorapidity ranges in minimum bias (top row) and dijet (botom row) event selections, with the data compared to different MC predictions [5].
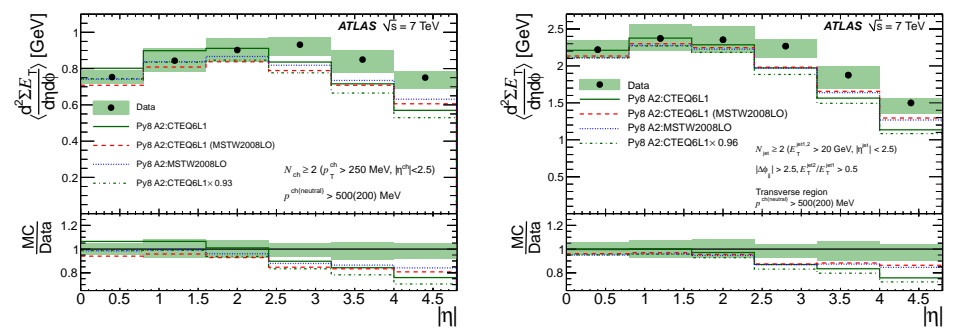

Figure 5: The distribution of transverse energy flow as a function of pseudorapidity are shown in minimum bias (left) and dijet (middle) event selections, with the data compared to the same tune but changing the PDF [5].

[8] The ATLAS Collaboration, Summary of ATLAS Pythia 8 tunes, ATL-PHYS-PUB-2012-003

[9] T. Sjostrand, S. Mrenna, and P. Skands, A brief introduction to Pythia 8.1, Comput. Phys. Commun. 178 (2008) 852-867, arXiv: 0710.3820 [hep-ph] .

[10] Pumplin, J. and others, New generation of parton distributions with uncertainties from global QCD analysis, JHEP 07 (2002) 012, arXiv: hep-ph/ 0201195.

[11] A. D. Martin, W. J. Stirling, R. S. Thorne and G. Watt, Eur. Phys. J. C 63, 189 (2009) [arXiv:0901.0002 [hep-ph]]. 